\title{
La Educación Artística como garantía de la inclusión educativa en Educación Primaria. Estudio comparado entre los currículums de Irlanda y España
}

\author{
Cabadas Maldonado, Virginia \\ Universidad Autónoma de Madrid, Madrid, España \\ virginia.cabadas@estudiante.uam.es
}

\section{Resumen}

Este trabajo busca abordar una comparación y análisis de los currículums de Educación Artística en la etapa de Educación Primaria de los países europeos de Irlanda y España, con el objeto de valorar si contribuyen o no a garantizar y fomentar la inclusión, equidad y justicia social en las aulas. Veremos $\mathrm{si}$, en el presente contexto de internacionalización de las políticas y tendencias de reforma educativas, son aun reconocibles las características propias de las tradiciones culturales y educativas de un país mediterráneo como es España y otro de tradición anglosajona como es el caso de Irlanda. Para la consecución de este objetivo general se utilizará una aproximación comparada como metodología básica de trabajo y se definirán los indicadores de comparación a partir de las siguientes dimensiones generales: objetivos, criterios de evaluación, número de descriptores temáticos, guías del profesorado, etc. Una vez establecidas las convergencias y divergencias entre las dos propuestas curriculares objeto de estudio, se concluirá con una reflexión acerca del potencial que la Educación Artística tiene, desde sus distintas vertientes, para abordar y trabajar por la inclusión y equidad educativa en las escuelas.

\section{Abstract}

In this paper the Arts Education curriculums in Primary Education of the European countries of Ireland and Spain are compared and analysed to see whether they contribute to ensure and encourage inclusion, equity and social justice in the classrooms. We will see if, the own characteristics of the cultural and educational traditions of a Mediterranean country such as Spain and another of Anglo-Saxon tradition as is the case of Ireland, are still recognizable in the present context of the policies and educational reforms tendencies internationalisation. To achive this general objective, a comparative approach will be used as the basic work methodology and the comparison indicators will be defined from the following general dimensions: objectives, evaluation criteria, number of thematic descriptors, teachers' guides, etc. Once established the convergences and divergences between the two curricular proposals object of study, it will conclude with a reflection about the potential that Arts Education has, from its different aspects, to address and work for inclusion and educational equity in schools.

Palabras clave: Educación Artística, Educación Primaria, currículum, inclusión, equidad, Irlanda, España.

Keywords: Arts Education, Primary Education, curriculum, inclusion, equity, Ireland, Spain.

\section{INTRODUCCIÓN}

A continuación, se presenta un análisis comparativo entre los países de Irlanda y España con el fin de observar la presencia o ausencia de referencias a la Educación Artística (a partir de ahora EA) como garantía o vía para abordar la inclusión educativa. Para ello, se han analizado diversas fuentes primarias y secundarias, con el objetivo de dar forma al marco teórico en relación con la Educación Artística inclusiva, así como el posterior análisis de los elementos encontrados en ambos currículums en relación con la existencia de convergencias o divergencias entre ambos. Para ello, el estudio utiliza como metodología una aproximación comparada y seguirá el diseño y desarrollo planteado por Caballero, Manso, Matarranz, y Valle, (2016). 
El motivo principal por el que se investiga este asunto es destacar la importancia que el área de EA tiene para el desarrollo infantil, en especial para el desarrollo social, emocional y personal, y justificar sus beneficios para la creación de un ambiente inclusivo donde se favorezcan las relaciones interpersonales e intrapersonales del alumnado, así como se tengan en cuenta sus distintas necesidades.

Las principales fuentes de consulta han sido los currículums para la Educación Primaria de ambos países, y en concreto la parte que se refiere al área de EA. Por un lado, el currículum de Irlanda fue elaborado por el National Council for Curriculum and Assesment (NCCA) en el año 1999 tras revisar el currículum anterior de 1971, un cuerpo estatutario del Departamento de Educación y Habilidades de la República de Irlanda que se encarga de aconsejar al Ministerio de Educación y Ciencia en relación con el currículum y la evaluación. El currículum se divide en lo que podríamos traducir como objetivos, conceptos y habilidades a desarrollar, contenidos, criterios de evaluación, y estándares de aprendizaje. Además, el NCCA lo complementa con otros materiales como guías para padres y guías para el profesorado. Por otro lado, en 2014, el Consejo de Ministros aprobó el currículum español para la Educación Primaria con base en la Ley Orgánica para la Mejora de la Calidad Educativa (2013), dividido en objetivos generales, contenidos, criterios de evaluación y estándares deaprendizaje.

Por consiguiente, no se busca analizar los cambios acontecidos en el tiempo sino una realidad estática presentada en estos dos documentos, uno más longevo que otro. Tras su comparación, se pretenden encontrar evidencias que aseguren la inclusión en las aulas y la EA como vehículo de garantía de la misma.

\section{EDUCACIÓN ARTÍSTICA INCLUSIVA}

Henderson y Lasley (2014) describen en uno de sus estudios las características que ha de tener una clase de arte para considerarse un ambiente inclusivo. Los autores lo enfocan a la etapa de Educación Infantil, las cuales podrían trasladarse a la etapa de Educación Primaria, objeto de esta investigación. Estos, nos dicen que para poder crear una clase inclusiva desde la EA se debe favorecer la existencia de diez áreas:

- Unidad o compañerismo, donde todos los estudiantes son tratados en equidad y se trabaja en equipo.

- Diversidad entre el alumnado.

- Construcción de comunidad a través del desarrollo de relaciones auténticas entre todas las personas (p. e., profesorado, familias, estudiantes), donde exista un clima de seguridad.

- Habilidades distintas entre el alumnado, donde cada estudiante es único.

- Actitud de «poder hacer», de que todo el alumnado es capaz si se atienden sus necesidades.

- Centrarse en el alumno, en sus habilidades, necesidades, intereses y estilos de aprendizaje.

- Espacio y tiempo compartido, con otras áreas, otros profesores, existencia de flexibilidad.

- Colaboración profesional, entre profesores, especialistas, etc.

- Documentación del proceso de aprendizaje del alumnado, entendido como evaluación de dicho proceso.

- Participación de las familias en la vida escolar.

Otros autores hablan también del arte haciendo referencia a sus beneficios para la inclusión social. Por ejemplo, García Morales (2012) defiende que el «arte puede ayudar a las personas en riesgo o en exclusión social a una integración más real y profunda, desarrollando en ellas tanto habilidades sociales, corporales y psicológicas, así como un desarrollo verdadero de la autoestima y el autoconcepto».

Por otro lado, Duncan (2007) habla de la posibilidad que ofrece el arte como entorno seguro donde la abstracción artística facilita la búsqueda y el ensayo de soluciones en un espacio donde poder expresarse con seguridad. A lo que un reciente informe de la Fundación Botín y la Universidad de Yale (2014) añade que «las artes proporcionan un marco donde poder estudiar las emociones con una mayor seguridad psicológica» (p. 20) de manera que hablan de la EA como un entorno donde poder crear, experimentar y aprender de manera segura sin temor al error. Además, el arte ofrece 
una vía de comunicación distinta al lenguaje oral: el lenguaje plástico, musical, corporal; y por tanto, ofrece al alumnado otras oportunidades de expresarse y comunicarse que fomentan la inclusión de todos los estudiantes (Domínguez Toscano, 2013; Duncan,2007).

Por otra parte, Pérez Barba (2010) defiende que «a través de la terapia artística se consigue superar las dificultades de adaptación», de modo que la EA puede contribuir a garantizar la inclusión del alumnado ya que, según Julie Allan (2014), las artes atraen a la gente a participar de ellas.

Finalmente, Bamford (2012) defiende que todos los niños con independencia de sus habilidades, capacidades, motivación inicial, etc., deberían poder recibir una formación en arte, cultura y creatividad como garantía de la inclusión y la igualdad educativa.

Dicho esto, ¿promueven y/o garantizan los currículums de Educación Artística para la etapa de Educación Primaria de la República de Irlanda y de España una educación inclusiva a través de la Educación Artística? ¿En qué medida se parecen o se diferencian ambos países en términos de Educación Artística?

\section{ANÁLISIS COMPARATIVO DE LOS CURRÍCULUMS DE IRLANDA Y ESPAÑA}

\subsection{Currículum irlandés de Educación Primaria}

El currículum irlandés actual para la Educación Primaria se extiende por un periodo de 8 años, desde lo que en España sería la etapa de Infantil (Junior and Senior Infants) hasta $6^{\circ}$ de Primaria. Dentro de sus tres propósitos generales de etapa podemos encontrar el objetivo de «capacitar al niño para que viva una vida plena como niño y tener en cuenta su potencial como un individuo único» (NCCAc, 1999, p. 7), de manera que se contempla una educación que respete la diversidad del alumnado en toda la etapa.

Se pone en relieve constantemente la necesidad de respetar y tolerar la diversidad cultural, religiosa, social, ambiental y étnica que existe en la sociedad global en la que vivimos, y señala que, pese a la presencia mayoritaria de la religión cristiana y su obligatoriedad en el currículum, en la sociedad irlandesa se reconocen todo tipo de creencias, valores y aspiraciones de otras religiones. En una sociedad donde el $96 \%$ de sus colegios públicos son religiosos, y un $90 \%$ de ellos católicos y muchos diferenciados por sexo, se reclama una educación no estereotipada en cuanto al género, sino inclusiva e igualitaria.

Antes de entrar en profundidad a analizar el currículum de Educación Artística, conviene mencionar algunas premisas, propósitos y objetivos que el currículum irlandés señala como prioritarios y que están estrechamente relacionadas con la inclusión escolar:

- El alumnado aprenderá trabajando en equipo y respetando las ideas de los demás

- La importancia del entorno como fuente de aprendizaje

- Capacitar al estudiante para explorar sus sentimientos, el mundo, etc.

- Capacitar al alumnado para desarrollar el respeto por la diferencia cultural

- Apreciar el arte en todas partes

- Desarrollar una conciencia positiva sobre sí mismo (confianza en sí mismo, autoestima y autoexpresión), una sensibilidad hacia otras personas y un respeto por los derechos, puntos de vista y sentimientos de los demás

- El pluralismo, el respeto por la diversidad y la importancia de la tolerancia

- La atención al alumnado con necesidad especiales e igualdad en el acceso a la educación.

\subsubsection{Elementos comunes del currículum irlandés de EA}

En primer lugar, el currículum de la Educación Primaria, del año 1999, sugiere dar a la asignatura de EA un tiempo mínimo de 3 horas semanales de las 28 horas y 20 minutos que conforman la 
semana escolar. El currículum de Educación Artística está formado por tres libros que cubren las áreas de Artes Visuales, Música y Teatro, y en los cuales podemos destacar la presencia reiterada de diversos elementos que fomentan la inclusión y que parecen comunes a las tres áreas y entre los que se destacan:

- Diversidad: se celebra la diferencia, el sentido individual y único de cada estudiante, la diversidad de habilidades, el potencial de todo el alumnado, las necesidades especiales de cada uno. En esta diversidad se presentan también distintos materiales a poder usar, actividades diferentes, contenidos a elegir y que abren el abanico de posibilidades para poder atender mejor la diversidad del aula siendo un currículum flexible.

- Multiculturalidad: se estudia el arte promoviendo el conocimiento de distintas culturas. También se sugiere adaptar el currículum, por ejemplo, en Música, a las características del colegio y del aula, y por consiguiente de la diversidad del alumnado.

- Sentido de identidad personal: se habla de explorar sus sentimientos, ideas y experiencias; su vida personal; de la contribución de las tres áreas al desarrollo de la confianza en sí mismos y de la autoestima. Además, se trabaja el sentido de pertenencia a un grupo y a una comunidad al trabajar el entorno, la comunidad en la que viven, la música irlandesa. En el caso del Teatro, se enfatiza en el carácter reflexivo que deriva de su puesta en práctica.

- Se fomenta la participación de todo el alumnado.

- Todas las áreas trabajan el desarrollo personal, social, emocional e intelectual del alumnado, de manera que contribuyen a mejorar las relaciones con los demás, promoviéndose así el trabajo en equipo y el compañerismo, en un sentido de compartir el trabajo con otros, conocer a los compañeros, trabajar la empatía, cooperar y colaborar como parte de un grupo.

Finalmente, en todas las áreas se habla de evaluación, la cual permite al docente detectar las necesidades del alumnado y adaptarse a ellas. Esta es continua y se hace, sobre todo, a través de la observación, tareas diseñadas en torno a la diversidad del aula y porfolios que deben revisarse con el alumnado.

\subsubsection{Guías para el profesorado}

Las guías para el profesorado desarrollan y hacen hincapié en lo anteriormente mencionado. Además, ofrecen recursos, estrategias, ejemplos de actividades, consejos, etc. a los docentes para desarrollar cada una de las áreas, sobre todo teniendo en cuenta que no existen especialistas por áreas. Por otro lado, insisten mucho en atender las necesidades del alumnado y entre otras cosas, se pide al profesorado:

- Involucrar a las familias: en las tres áreas se destaca la labor de los padres en el apoyo a sus hijos en relación con sus distintas habilidades en las artes, así como a su participación en la vida del centro.

- Involucrar a la comunidad: sobre todo en Plástica desde la visita a museos y galerías, y en Música a través de trabajar con la radio, la televisión, organizaciones musicales.

- Trabajar con la diversidad: en Música y en Teatro se destacan los talleres multinivel y sus beneficios para el proceso de aprendizaje; en Música también se dan multitud de ejemplos para trabajar distintas actividades con personas con diversidad funcional. Sobre todo, se enfatiza la necesidad de adaptar y crear distintas actividades, materiales, espacios o agrupaciones según las distintas necesidades de los estudiantes, se destaca el diálogo del docente con el estudiante acerca de sus trabajos.

- Flexibilizar el currículum, y por tanto esa adaptación es posible, de manera que la planificación dependerá de las circunstancias, aptitudes, intereses, etc. del alumnado, y se beneficiará de oportunidades que surjan de manera espontánea.

- Garantizar el acceso igualitario de todos y todas a los recursos, materiales y asignaturas, donde además se promueva un clima de aula de aceptación, apoyo y respeto hacia los demás y sus trabajos. 
- Partir de las experiencias del alumnado, teniéndose en cuenta en Música lo que ya saben, en Plástica sus habilidades naturales o estadios de desarrollo, y en Teatro sus propias vidas (conflictos internos, preocupaciones, etc.).

\subsubsection{Guías para los padres}

Estas guías se ofrecen bajo la premisa del rol central que tienen estos en la educación de sus hijos. En las mismas se defiende la necesidad de que exista un fuerte vínculo entre los padres, la escuela y el profesorado para conseguir un buen desarrollo del alumnado, y a quienes invitan a participar en la vida del centro, por ejemplo, organizando eventos artísticos.

\subsection{Currículum español de Educación Primaria}

El currículum español abarca un periodo de seis cursos. Entre sus objetivos generales de etapa cabe destacar el fomento del:

- Pluralismo.

- Respeto a los demás.

- Trabajo en equipo.

- Actitudes de confianza en uno mismo.

- Habilidades de resolución de conflictos.

- Actitudes de no violencia y no discriminación de ningún tipo, evitando los estereotipos y prejuicios.

- Respeto a la diversidad: cultural, de género, etc.

- Habilidades socioemocionales.

Además, se habla también de atender de manera individualizada y personalizada al alumnado, fomentar la equidad, calidad e inclusión educativa de personas con diversidad funcional y especialmente, tener en cuenta los ritmos y estilos de aprendizaje de cada estudiante. Este currículum se muestra también flexible desde el punto de vista de que puede ser adaptado y completado a las características de los alumnos.

\subsubsection{Elementos comunes del currículum español de EA}

En primer lugar, conviene comentar que la EA ha pasado a un segundo plano en el currículum español en los últimos 30 años pues su presencia se ha visto reducida en el currículum de la etapa. En la Tabla 1 se puede ver el ejemplo de la Comunidad Autónoma de Madrid (C.M.) donde, desde la LOGSE (1990) hasta la última ley educativa, la LOMCE (2013), la EA se ha reducido en un $50 \%$ en el currículum escolar de esta comunidad autónoma. En España fijar el horario lectivo de las asignaturas específicas, bloque al que pertenece la EA, es competencia de las Comunidades Autónomas por lo que puede variar de unas comunidades a otras. La semana escolar en España es de 25 horas.

Tabla 1. Evolución del $n^{\circ}$ de horas semanales de EA en la Comunidad de Madrid.

\begin{tabular}{|c|c|c|c|c|c|}
\hline \multicolumn{6}{|c|}{ Número de horas semanales de Educación Artística (C.M.) } \\
\hline $1^{0}$ & $2^{0}$ & $3^{0}$ & $4^{0}$ & $5^{0}$ & 6 \\
\hline 1,5 & 1,5 & 1,5 & 2,5 & & \\
\hline
\end{tabular}


Además, como se puede leer también en la LOMCE (art. 6), la EA no tiene un carácter obligatorio en el currículum y por consiguiente no queda garantizada su enseñanza en las escuelas. De esta manera, se abre la puerta a los centros para poder decidir si incluir entre su oferta esta asignatura o prescindir de ella.

El currículum español de EA se divide en dos partes: Educación Plástica y Educación Musical, quedando excluidas otras áreas específicas como el Teatro. Del análisis del currículum se deducen los siguientes elementos como características de una EA inclusiva en España:

- Carácter multiexpresivo y comunicativo: la EA se presenta como un medio de comunicación y expresión de ideas, sentimientos y pensamientos inherente al ser humano, donde especialmente se desarrollan el lenguaje plástico y musical, y la expresión corporal como parte de este último; y que se presenta por lo tanto como una vía de comunicación distinta al lenguaje oral y escrito. En la Educación Plástica se sugieren diversos modos de expresión, a través del cómic, el cine, la fotografía, es decir, los medios audiovisuales, así como el dibujo, y otros materiales no específicos.

- Diversidad: En Educación Plástica, el alumnado debe utilizar distintos materiales y las técnicas más adecuadas y que mejor se adapten a sus necesidades.

- Multiculturalidad: se pretenden analizar, por ejemplo, imágenes de distintos contextos culturales e históricos, o en el caso de Música, se hace especial hincapié en el estudio de obras musicales, canciones, danzas, etc. nacionales y de otros lugares resaltándose la necesidad de valorar la aportación multicultural al enriquecimiento personal, social y cultural.

- Conocimiento del Patrimonio cultural: ambos bloques ponen el énfasis en el conocimiento y disfrute del patrimonio cultural español, entendiendo el mismo como el legado cultural que recibimos de nuestros antepasados, que nos conecta con ellos y que genera un vínculo entre individuos (de los Reyes, 2009). De esta definición y al resaltarse también el uso del entorno próximo e imaginario, podemos deducir el objetivo de crear un sentido de pertenencia a la comunidad a través de la EA en general.

- Trabajo en equipo: este se fomenta en ambas áreas y se acentúa el respeto a las ideas de los demás.

\section{3. ¿Dónde se encuentran y dónde se separan amboscurrículums?}

Con base en lo expuesto anteriormente, se puede observar una clara convergencia en muchos de los puntos que ambos currículums fomentan. Pese al volumen claramente desproporcionado de un currículum y otro, en ambos se refleja una preocupación por la inclusión educativa desde la etapa en general, desde donde se atienden en todas las áreas las necesidades educativas, la diversidad del alumnado en todas sus facetas, donde se tienen en cuenta las habilidades socioemocionales del alumnado, su sentido de individuo único, su autoestima y autoexpresión, el trabajo en equipo y el respeto hacia los demás y sus ideas.

Por otro lado, dentro del área de EA la principal diferencia entre ambos países reside en la falta del Teatro como uno de los bloques del currículum español, lo que conlleva a España a perder algunos de los beneficios anteriormente mencionados de los que Irlanda sí se nutre. En lo que respecta a la Música y las Artes Plásticas y Visuales, ambos países destacan el respeto a la diversidad, la multiculturalidad, el desarrollo de un sentido de pertenencia a una comunidad, el trabajo en equipo, la igualdad de oportunidades y el respeto a los demás como elementos de inclusión que se consiguen a través de la EA.

Finalmente, el currículum irlandés cuenta además con otro material complementario, las guías para padres y para el profesorado, que rematan esta lista incluyendo, además de otros elementos comunes al currículum español, la involucración de las familias en la vida escolar, uno de los puntos que incluían Henderson y Lasley en su lista y que el currículum español apenas menciona. 


\section{DISCUSIÓN Y CONCLUSIONES}

Expuestas las convergencias y divergencias entre ambos currículums, se puede concluir que el área de EA sí garantiza la inclusión educativa en el currículum irlandés, aunque no se puede decir lo mismo del currículum español dado el carácter específico no obligatorio de la asignatura. En este sentido, se puede afirmar también que la EA en España, tal y como aparece reflejada en el currículum, fomenta la inclusión educativa pero no queda garantizada al existir la posibilidad de no llegar a ofertarse en un centro.

Tras el análisis anterior, se sugieren las siguientes propuestas de mejora:

\section{En el currículum español:}

- Incluir el Teatro como tercer bloque de la EA.

- Dotar de un carácter obligatorio a la EA.

\section{En el currículum irlandés:}

- Adaptar el material para que sea más manejable, evitando las repeticiones y reduciendo su volumen.

Este análisis comparativo expone las conclusiones extraídas exclusivamente al comparar los documentos de los currículums educativos de la etapa de Educación Primaria de los países europeos de Irlanda y España. De manera que, este estudio debería complementarse con una investigación de la realidad en las aulas de ambos países para ver en qué medida se fomenta la inclusión desde el área de EA.

\section{REFERENCIAS BIBLIOGRÁFICAS}

Allan, J. (2014). Inclusive Education and the Arts. Cambridge Journal of Education, 44(4), 511- 523.

Bamford, B. (2012). Eslabones de una cadena: aspectos que influyen en la enseñanza creativa. Fundación Botín. (Ed.), ¡Buenos días creatividad! Hacia una educación que despierte la capacidad de crear, 57-68. Recuperado de: https:// www.fundacionbotin.org/educacion-contenidos/investigacion-e-informes.html.

De Los Reyes Leoz, J. L., (2009). Del patrimonio cultural al museo infantil. Tarbiya: Revista de investigación e innovación educativa, 40, 107-124.

Department of Education and skills (s. f). Diversity of patronage. [sitio web] En https://www.education.ie/en/Schools-Colleges/Information/Diversity-of-Patronage/Diversity-of-Patronage-Survey-of-Parents.html.

Domínguez Toscano, P. M. (2013). Ser emocionalmente competentes, con arte. Educación artística terapéutica para la gestión emocional. Multiárea: revista de didáctica. Universidad de Castilla-La Mancha, 6, 19-121.

Duncan, N. (2007). Trabajar con las emociones en arteterapia. Arteterapia: papeles de arteterapia y educación artística para la inclusión social, 2,39-49.

España. Real Decreto 126/2014, de 28 de febrero, por el que se establece el currículo básico de la Educación Primaria, Boletín Oficial del Estado, 1 de marzo de 2014, Núm. 52, pp. 19349- 19420.

García Morales, C. (2012). ¿Qué puede aportar el arte a la educación? El arte como estrategia para una educación inclusiva. Arte y Sociedad. Revista de Investigación, 1.

Henderson, C. M. y Elizabeth, L. (2014). Creating Inclusive Clasrooms through the Arts. Dimensions of Early Childhood, 42(3), 11-17.

Ivcevic, Z., Hoffmann, J., Brackett, M. y Fundación Botín (2014). Artes, emociones y creatividad. Fundación Botín (Ed.) Arte y emociones que potencian la creatividad. Recuperado de: https://www.fundacionbotin.org/paginas-interiores-de-una-publicacion-de-la-fundacion-botin/artes-y-emociones-que-potencian-la-creatividad.html.

NCCA (1999a). Drama. Arts Education. Primary School Curriculum. Dublin: Government of Ireland Publications.

NCCA (1999b). Drama. Arts Education. Teacher Guidelines. Dublin: Government of Ireland Publications. 
NCCA (1999c). Introduction. Primary School Curriculum. Dublin: Government of Ireland Publications.

NCCA (1999d). Music. Arts Education. Primary School Curriculum. Dublin: Government of Ireland Publications.

NCCA (1999e). Music. Arts Education. Teacher Guidelines. Dublin: Government of Ireland Publications.

NCCA (1999f). Visual Arts. Arts Education. Primary School Curriculum. Dublin: Government of Ireland Publications.

NCCA (1999g). Visual Arts. Arts Education. Teacher Guidelines. Dublin: Government of Ireland Publications.

NCCA (1999h). Your child's learning. Guidelines for parents. Dublin: Government of Ireland Publications.

Pérez Barba, M. S. (2010). EnamorARTE. La arteterapia para la autoexpresión y el desarrollo integral. En González Vida, R., Moleón Viana, M. A. \& González Castro, C. (Eds.), I Congreso Internacional Arte, Ilustración y Cultura Visual en Educación Infantil y Primaria: construcción de identidades, 553-558. Granada, España. 